\title{
Neonatal intra-atrial baffle repair for isolated ventricular inversion with left isomerism: a case report
}

\author{
Yuta Kuwahara*, Yukihiro Takahashi, Yuya Komori, Naohiro Kabuto and Naoki Wada
}

\begin{abstract}
Background: Discordant atrioventricular connection with concordant ventriculoarterial connection, otherwise known as isolated ventricular inversion (IVI), is an extremely rare congenital cardiac malformation. Reports on the corrective surgery for this anomaly in neonates are few, and the procedure is difficult and complicated. Herein, we report our use of atrial septostomy as a palliative procedure followed by corrective surgery for the repair of neonatal IVI with situs ambiguous(inversus) morphology.
\end{abstract}

Case presentation: A 2-day-old girl weighing $3.5 \mathrm{~kg}$ was admitted to our hospital with a low oxygen saturation $\left(\mathrm{SpO}_{2}\right)$ of $70 \%$ She was diagnosed with IVI [situs ambiguous(inversus), D-loop, and D-Spiral], atrial septal defect, patent ductus arteriosus (PDA), interrupted inferior vena cava with azygos continuation to the left superior vena cava (SVC), and polysplenia by transthoracic echocardiography and cardiac computed tomography. We planned to perform corrective surgery and decided to first increase interatrial mixing by performing surgical atrial septostomy and PDA ligation 7 days after birth. However, despite the surgical septostomy, pulmonary venous blood flowed toward the right ventricle via the tricuspid valve rather than toward the left-sided atrium and hypoxemia persisted. We decided to perform the intra-atrial switch procedure at the age of 17 days via a re-median sternotomy. The cardiopulmonary bypass (CPB) circuit was established with ascending aorta and venous drainage through the SVC and hepatic veins. Utilizing a left-sided atrium(I-A) approach, a bovine pericardial patch was used for the intra-atrial baffle, which was trimmed into a trouser-shaped patch. Continuous suture using the patch was lying from the front of the right-sided upper pulmonary vein and rerouted SVC, hepatic vein, and coronary sinus to the tricuspid valve. Overall, CPB weaning proceeded smoothly; however, direct current cardioversion was performed for junctional ectopic tachycardia. The postoperative course was uneventful. Her postoperative $\mathrm{SpO}_{2}$ improved (approximately 99-100\%); overall, the patient showed clinical improvement. Discharge echocardiography showed normal biventricular function and an intact atrial baffle with no venoatrial or atrioventricular obstruction.

Conclusion: We successfully performed an intra-atrial switch procedure for isolated ventricular inversion in a neonate. Long-term follow-up will be necessary to ensure the maintenance of optimal cardiac function.

Keywords: Isolated ventricular inversion, Neonate, Intra-atrial baffle repair

*Correspondence: ykuwaha@shi.heart.or.jp

Department of Cardiovascular Surgery, Sakakibara Heart Institute, 3-16-1, Asahicho, Fuchu, Tokyo 183-0003, Japan

\section{Background}

Discordant atrioventricular connection with concordant ventriculoarterial connection, otherwise known as isolated ventricular inversion (IVI), is an extremely rare congenital cardiac malformation [1]. IVI results in parallel circulation, similar to transposition of the great arteries (TGA). Ventricular septal defect (VSD) is commonly 
associated with IVI. Without corrective surgery, survival beyond infancy is rare. Reports on the corrective surgery for this anomaly in neonates are few, and the procedure is difficult and complicated [2,3]. Consequently, the surgical strategy for neonatal IVI remains challenging, even more so when the atrial situs is ambiguous(inversus).

Herein, we report our use of atrial septostomy as a palliative procedure followed by corrective surgery for the repair of neonatal IVI showing situs ambiguous(inversus) morphology.

\section{Case presentation}

A 2-day-old girl who weighed $3.5 \mathrm{~kg}$ was admitted to our hospital because of low oxygen saturation $\left(\mathrm{SpO}_{2}\right)$. Her $\mathrm{SpO}_{2}$ was $70 \%$ and her previous doctor suspected total anomalous pulmonary venous return as the cause. She was diagnosed with IVI (situs ambiguous(inversus), D-loop, and D-Spiral), atrial septal defect (ASD), patent ductus arteriosus (PDA), interrupted inferior vena cava (IVC) with azygos continuation to the left superior vena cava (SVC), and polysplenia by transthoracic echocardiography and cardiac computed tomography (CT) (Fig. 1).

From admission, she had PDA-dependent hemodynamics, and she was started on lipo-prostaglandin E1 therapy. At the age of 5 days, she began developing congestive heart failure due to decreased pulmonary vascular resistance and the increased blood flow of the PDA. It became difficult to control her heart failure due to the PDA-dependent hemodynamics.

We believed that closure of the PDA would cause further hypoxia. We planned to perform corrective surgery, but very few case reports of an intra-atrial switch procedure for IVI in neonates are currently available. Therefore, we first considered increasing interatrial mixing, but because balloon atrial septostomy is difficult for an interrupted IVC, we performed surgical atrial septostomy and PDA ligation at the age of 7 days.

Despite the surgical septostomy, pulmonary venous blood flowed toward the right ventricle via the tricuspid valve rather than toward the left-sided atrium, and hypoxemia persisted. Various measures, such as nitric oxide administration and volume loading, were not effective for hypoxemia over several days. The $\mathrm{SpO}_{2}$ was around $60 \%$ and the partial pressure of arterial oxygen was around $30 \mathrm{mmHg}$, which was very low; hence, we decided to perform the intra-atrial switch procedure.

The surgery was carried out at the age of 17 days via a re-median sternotomy. The cardiopulmonary bypass (CPB) circuit was established with ascending aorta and venous drainage through the SVC and hepatic veins. Myocardial protection was provided with hypothermia and the administration of antegrade cold blood glucoseinsulin-potassium solution. A left-sided atrium(l-A) approach was employed and sufficient enlargement of the ASD was confirmed. Actually, both atria are left atrium in this case. The coronary sinus (CS) was cut back about $10 \mathrm{~mm}$. A bovine pericardial patch was used for the intra-atrial baffle, which was trimmed into a trousershaped patch [4]. (It was an unplanned reoperation and the condition of autologous pericardium was inadequate, so its use was abandoned.) The patch was attached with running 7-0 monofilament sutures. Continuous suture using the patch was lying from the front of the rightsided upper pulmonary vein and rerouted SVC, hepatic vein, and CS to the tricuspid valve. Hepatic vein passage was expanded with an additional patch to prevent stenosis. After the intra-atrial switch procedure, the left-sided atrium was closed (Fig. 2). Overall, CPB weaning proceeded smoothly; however, direct current cardioversion was performed for junctional ectopic tachycardia (JET). The CPB time was $99 \mathrm{~min}$, and the aortic cross-clamping time was $77 \mathrm{~min}$. We decided to delay sternal closure to deal with potential arrhythmias and low-output syndrome. The postoperative course was uneventful, and postoperative $\mathrm{SpO}_{2}$ was much improved (approximately 99-100\%). Discharge echocardiography taken after Mustard operation showed that the systemic venous flow routed to the right-sided atrium $(\mathrm{r}-\mathrm{A})$ through the systemic venous baffle, whereas the pulmonary venous flow was routed to l-A through the pulmonary venous baffle. And this echocardiography showed normal biventricular function and an intact atrial baffle with no venoatrial or atrioventricular obstruction (Fig. 3). Accordingly, the patient underwent the Mustard procedure and clinical improvement was obtained.

\section{Discussion}

In 1966, Van Praagh proposed the term IVI to refer to the rare cardiac morphology of ventricular inversion without TGA [5]. Since then, there have been very few reports of radical surgery for IVI in neonates. Herein, we report a case of neonatal IVI with situs ambiguous(inversus) morphology and heart failure. The patient developed hypoxemia after PDA ligation and atrial septostomy, and eventually underwent intra-atrial baffle repair (Mustard surgery), which resulted in a favorable outcome.

The present case suggested important feature of this disease. Existence of VSD or PDA greatly influence its clinical course.

First, in this case, VSD, which is often associated with this disease, was not observed. Lack of interventricular mixing resulted in poor oxygenation.

Arciprete and colleagues report cases of IVI with VSD (PDA absent in all cases) in which radical surgery was performed after the neonatal period. They also report a case of radical surgery being required for a newborn with 

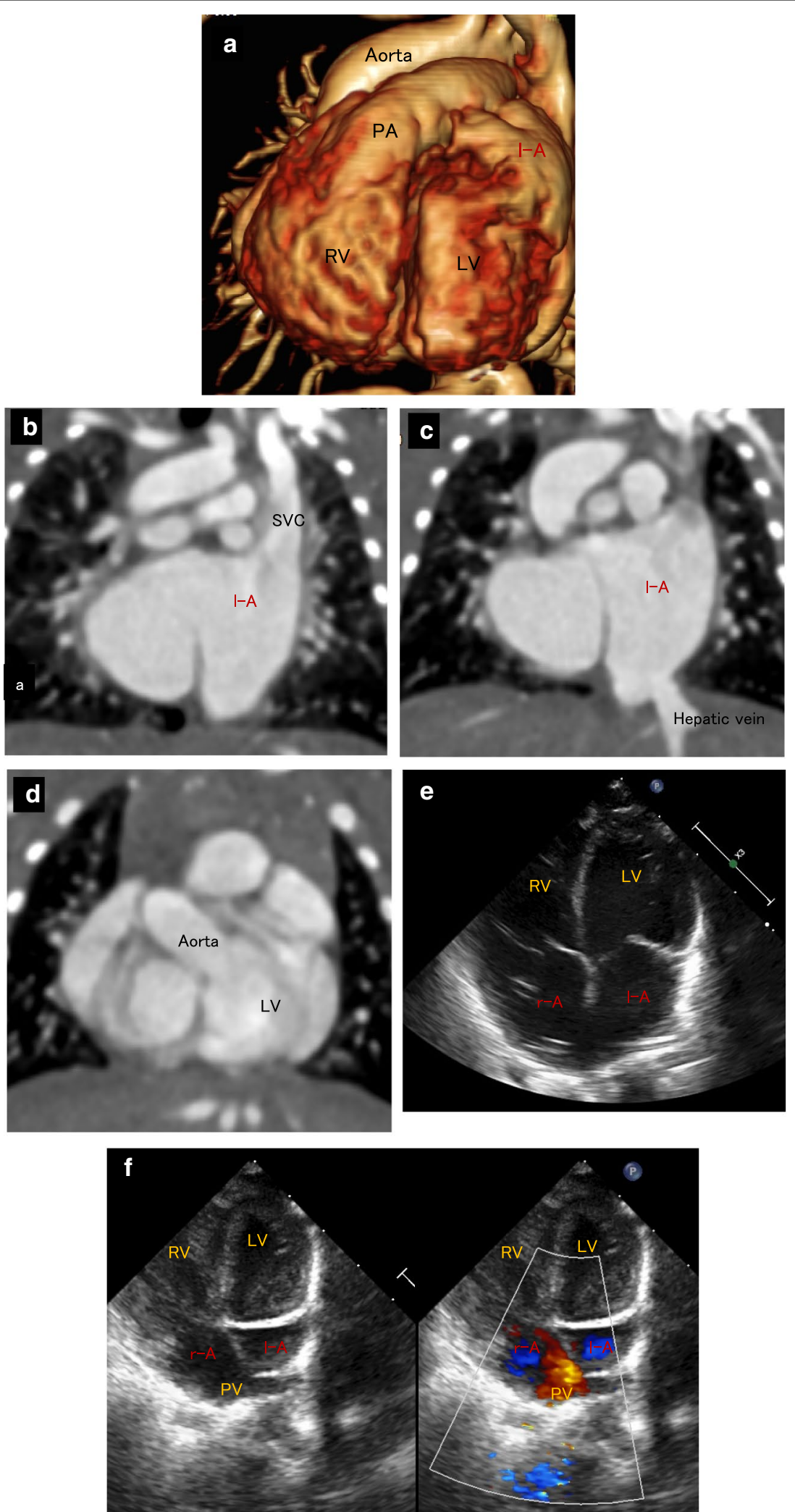

Fig. 1 The computed tomography scans (a-d), and the transthoracic echocardiography $(\mathbf{e}, \mathbf{f})$ obtained from the patient. These figures indicate situs ambiguous(inversus), D-loop, and D-Spiral, atrioventricular discordance, ventriculoarterial concordance 


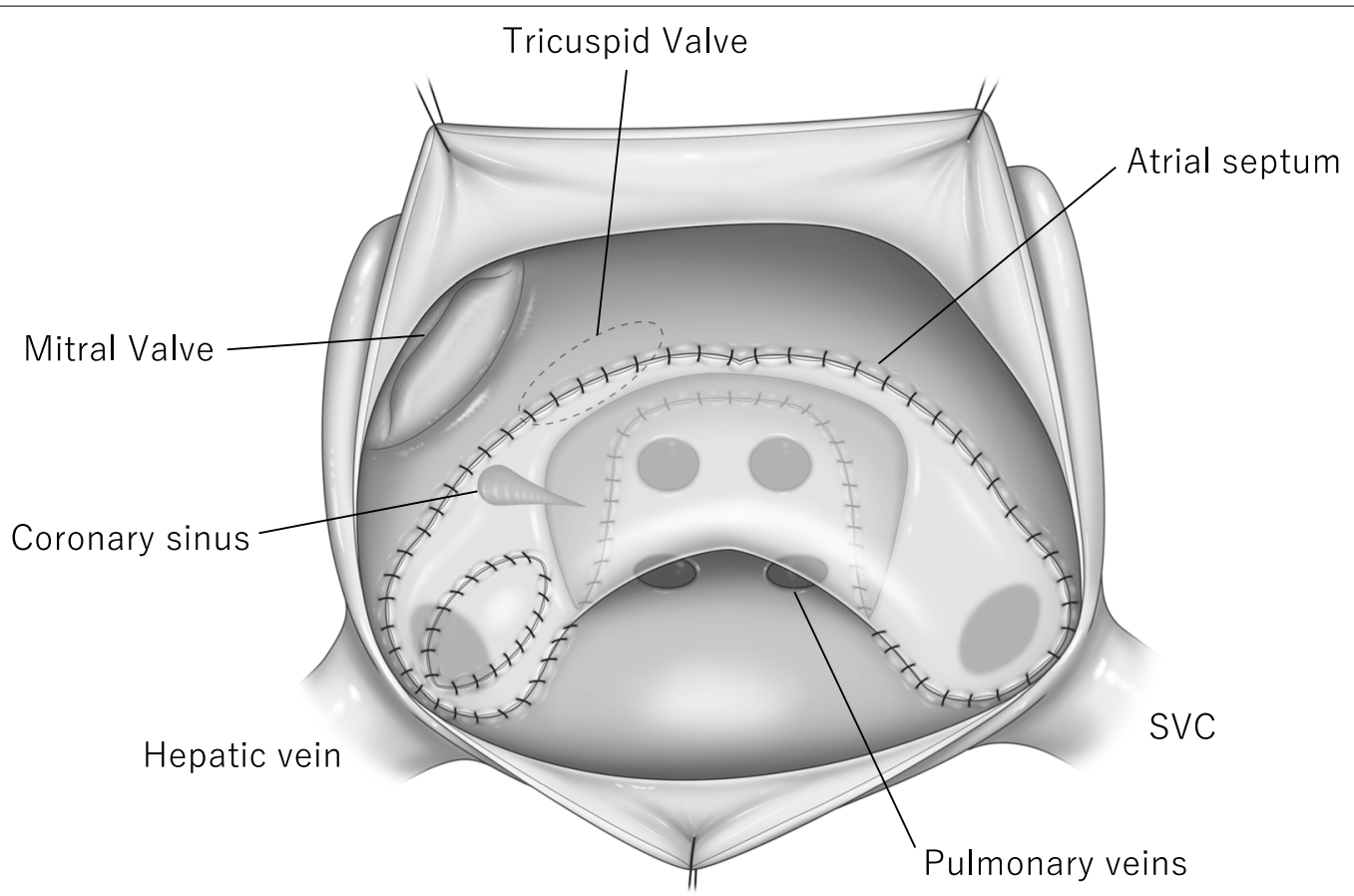

Fig. 2 Surgical schema of intra-atrial baffle repair viewed through a left-sided atriotomy

IVI without VSD (surgically undetectable) [6]. Furthermore, when PDA co-exists with VSD, early intervention due to the high pulmonary blood flow is required $[7,8]$. Thus, radical neonatal surgical intervention may be less necessary when PDA is absent and VSD exists. In addition, IVI often coexists with left atrial isomerism.

Left atrial isomerism is associated with multiple systemic and pulmonary venous abnormalities. Partial anomalous pulmonary venous connection or total anomalous pulmonary venous connection may increase oxygen levels in these patients [3].

Second, the patient in this case had PDA-dependent hemodynamics. We think that this is an important point that influenced our treatment strategy. In the present case, heart failure developed gradually from the age of 5 days. The cause was thought to be high pulmonary blood flow due to PDA, and we found that the PDAdependent circulation was difficult to manage. Therefore, we performed PDA ligation and atrial septostomy, but it was difficult to achieve adequate atrial mixing as in type I TGA. Although Mauri and colleagues described that atrial septostomy was effective for the cyanosis of IVI [9], it may not always lead to improvement of oxygenation. Moreover, a ridge-like structure was observed immediately above the CS by ultrasonography during the course, indicating the anatomical feature that was likely guiding the pulmonary vein blood flow toward the tricuspid valve.
If the PDA had not been closed in this case, oxygenation could have been maintained, but heart failure would have progressed. If VSD is present, a strategy to close the PDA may be appropriate. Thus, closure of the PDA favors heart failure, but adversely affects the oxygenation level.

Another important point of this case was actual diagnosis of IVI. Because this case was diagnosed as polysplenia, it seemed to be difficult to give precise diagnosis of atrioventricular discordance. Although some reports have excluded cases of atrial situs ambiguous, others have included cases that were not defined as atrial situs solitus. Further, interestingly, all cases without ventricular septal defect and atrioventricular septal defect in the past reports of IVI showed an azygos or a hemi azygos connection [3]. In this disease, many cases have shown left isomerism and azygos or hemiazygos connection. Further, it may be difficult to distinguish the right and left atrium morphologically. In fact, defining atrial situs solitus does not seem easy. These issues are controversial among cardiac morphologists [10]. Further embryologi$\mathrm{cal}$ and anatomical research is warranted in the future.

To determine the optimal treatment strategy for IVI in the neonatal period, it is necessary to consider the degree of heart failure and hypoxemia after appropriately evaluating any coexisting malformations (especially VSD and PDA). Although radical surgery by an atrial switch procedure is feasible in the neonatal period, as in the present case, the difficulty of radical surgery is thought to 

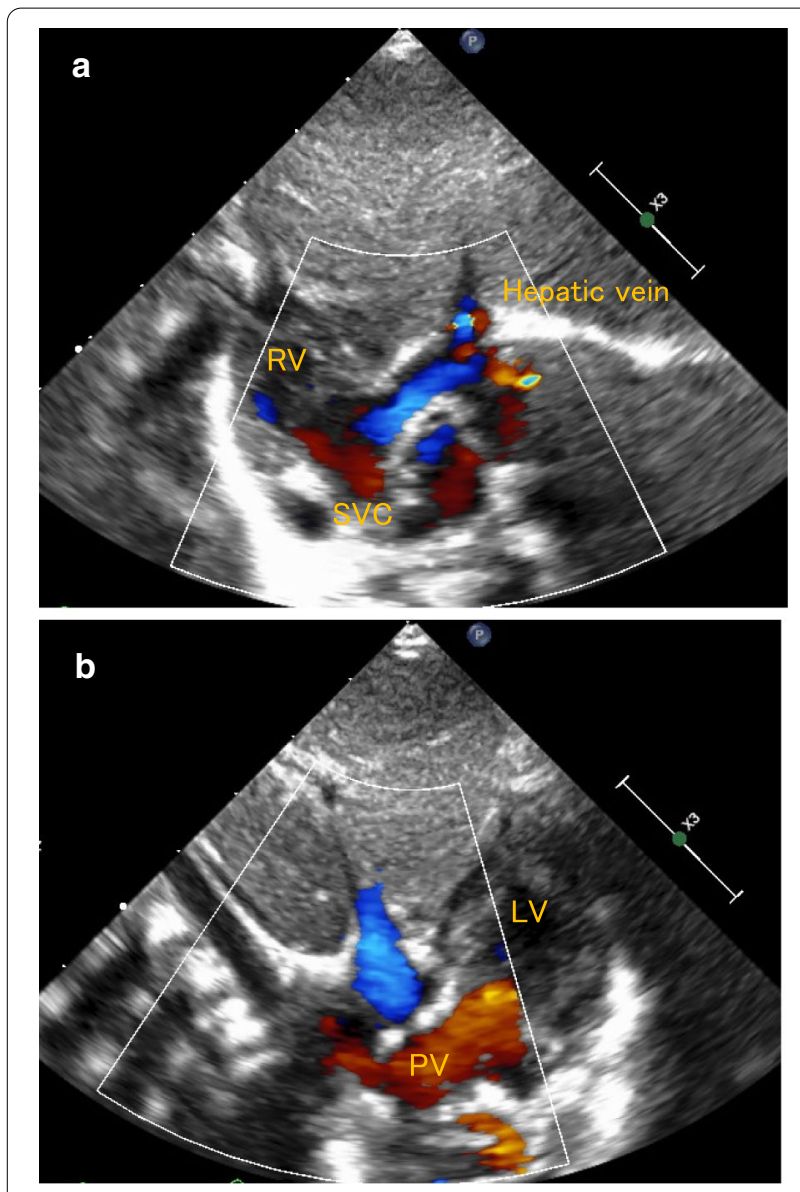

Fig. 3 a, b The transthoracic echocardiography taken after Mustard operation showed that the systemic venous flow routed to the right-sided atrium through the systemic venous baffle, whereas the pulmonary venous flow was routed to I-A through the pulmonary venous baffle

decrease with the physical growth of the patient. Careful follow-up with attention to potential venous stenosis in the atrium and arrhythmias in the long term is necessary in these patients.

\section{Conclusion}

In conclusion, we successfully performed an intra-atrial switch procedure for isolated ventricular inversion in a neonate. Long-term follow-up will be necessary to ensure the maintenance of optimal cardiac function.

\section{Abbreviations}

IVI: Isolated ventricular inversion; VSD: Ventricular septal defect; SpO2: Oxygen saturation; ASD: Atrial septal defect; PDA: Patent ductus arteriosus; IVC: Inferior vena cava; SVC: Superior vena cava; CT: Computed tomography; CPB: Cardiopulmonary bypass; CS: Coronary sinus; JET: Junctional ectopic tachycardia; TGA:Transposition of the great artery; r-A: Right-sided atrium; I-A: Left-sided atrium.

\section{Acknowledgements}

Not applicable.

\section{Authors' contributions}

NW and YT conceived this case presentation and drafted the manuscript. All authors read and approved the final manuscript.

\section{Funding}

None of the authors received any funding.

\section{Availability of data and materials}

Data sharing is not applicable to this article as no datasets were generated or analyzed during the current study.

\section{Ethics approval and consent to participate}

Not applicable.

\section{Consent for publication}

Informed consent was obtained from the patient for publication of this case report.

\section{Competing interests}

The authors declare that they have no competing interests.

Received: 31 May 2020 Accepted: 21 September 2020

Published online: 29 September 2020

\section{References}

1. Ranjit MS, Wilkinson JL, Mee RBB. Discordant atrioventricular connexion with concordant ventriculo-arterial connection(so-called isolated ventricular inversion) with usual atrial arrangement(situs solitus). Inter J Cardiol. 1991a;31:114-7.

2. Murat $\mathrm{S}$, Aysel T, Ersin E. A rare indication for senning operation: isolated ventricular inversion and ventricular septal defect. Anatol J Cardiol. 2015;15:E21.

3. Doff $B M$, Mohan $\mathrm{R}$, Norman $\mathrm{H}$, et al. Intraatrial baffle repair of isolated ventricular inversion with left atrial isomerism. Ann Thorac Surg. 1996;62:1529-32.

4. Stark J. Concordant transposition-Mustard operation. Surgery for congenital heart defects, $1^{\text {st }}$ edition. Grune and Stratton, 1983.

5. Van Praagh R, Van Praagh S. Isolated ventricular inversion: a consideration of the morphogenesis, definition and diagnosis of nontransposed and transposed great arteries. Am J Cardiol. 1966;17:395-406.

6. Arciprete P, Macatney FJ, de Leval M, et al. Mustard's operation for patients with ventriculoarterial concordance: report of two cases and a cautionary tale. Br Heart J. 1985;53:443-50.

7. Pasquini L, Sanders SP, Parness I, et al. Echocardiographic and anatomic findings in atrioventricular discordance with ventriculoarterial concordance. Am J Cardiol. 1988;62:1256-62.

8. Ranjit MS, Wilkinson JL, Mee RB. Discordant atrioventricular connexion with concordant ventriculo-arterial connexion (so-called "isolated ventricular inversion") with usual atrial arrangement (situs solitus). Int J Cardiol. 1991b;31:114-7.

9. Mauri L, Christopher L, Elliott S, et al. A rare congenital cardiac malformation with situs inversus and discordant atrioventricular and concordant ventriculoarterial connections: dignosis and surgical treatment. Am Heart J. 1981;101:355-6.

10. Uemura H, Ho SY, DeVine WA, Kilpatrick LL, Anderson RH. Atrial appendages and venoatrial connectionsin hearts from patients with visceral heterotaxy. Ann Thorac Surg. 1995;60:561-9.

\section{Publisher's Note}

Springer Nature remains neutral with regard to jurisdictional claims in published maps and institutional affiliations. 